\title{
The analysis of dynamic response of rock mass around tunnel under dynamic unloading
}

\author{
Xian $\mathrm{Li}^{1, \mathrm{a}}$ \\ ${ }^{1}$ Faculty of Civil Engineering and Mechanics, Kunming University of Science and Technology, \\ Kunming, China \\ aemail: demonjjun@gmail.com
}

Keywords: Dynamic unloading; dynamic response; circular tunnel; elastic area.

Abstract. During the excavation of rock mass with the method of drill and blast, the release of in-situ stress, also known as excavation load on boundary, is traditional a dynamic process, so the inertia and all other dynamic responses induced by the release of in-situ stress cannot be ignored. Compared with corresponding quasi-static unloading, dynamic displacement field of surrounding rock is decomposed into the quasi-static displacement and the dynamic displacement. Disturbance in the surrounding rock increases with a decrease in unloading rate.

\section{Introduction}

In rock blasting excavation of underground projects, unloading relaxation (stress redistribution) process caused by the excavation traditionally lasts for a long time [1]. The throwing motion time is about a millisecond to a few hundred milliseconds scale. So, the release of in-situ stress can be seen as a dynamic process. Lu et al. [2] employed the theory of stress wave and propagation to analyze the movement process of the rock block under the sudden unloading of the initial stress during rock excavation by blasting. Yi et al. [3] used numerical method to simulate the high dynamic unloading of initial stress during rock excavation. Ren et al. [4] first make real-time CT testing of the meso-damage evolution law of the whole failure process of rock in unloading condition using the specified loading equipment corresponding to the CT machine developed by the authors in rock mechanics field.

The indoor test of unloading rate is often low. So, it is cannot correctly simulated the transient unloading effect in the process of rock damage on the surface of the situation. We conducted this study to show the detailed process of release of hydrostatic in-situ stress accompanying dynamic response of the surrounding rock.

\section{Model Establishment}

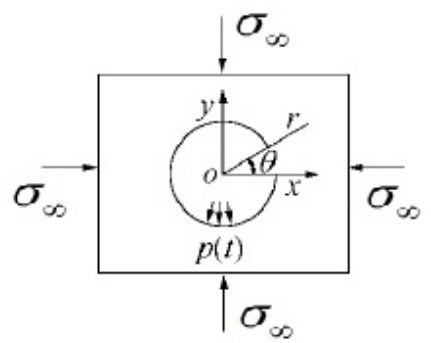

(a)

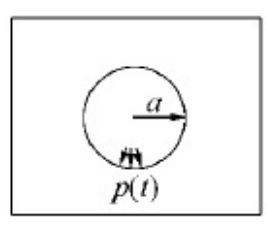

(b)

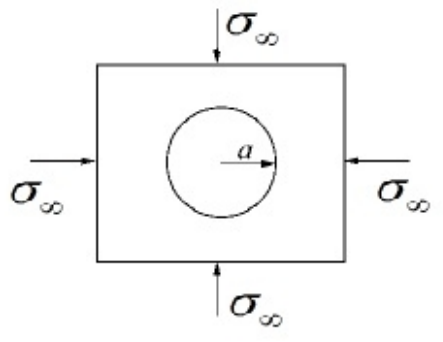

(c)

Fig. 1: The original problem is divided into two sub-problems: (a) the original problem; (b) the first sub-problem; (c) the second sub-problem 


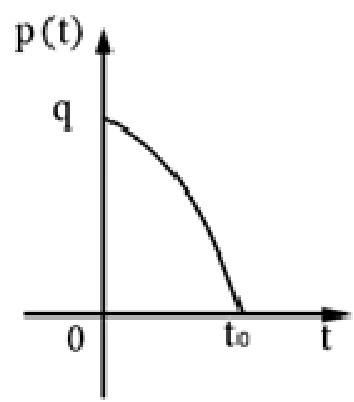

Fig. 2: Function relationship between load and time during excavation process

It is shown from Figure 1 that the circular tunnel is subjected to hydrostatic in situ stress $\sigma_{\infty}$ at infinity. As a results, the dynamic problem can be divided into two sub-problems as shown in Figure 1(b)-(c).

As shown in Figure 2, a dynamic pressure can be written as:

$$
P(t)=f t^{2}+g t+h
$$

where, $f=-\frac{\sigma_{\infty}}{t_{0}^{2}}$, and $t_{0}$ is complete time of excavation process; $g=0 ; h=\sigma_{\infty}$.

Excavation of the circular tunnel is axisymmetric problem. Under axisymmetric conditions, the stress-strain relations of rock masses is

$$
\begin{aligned}
& \sigma_{r}=c_{11} \varepsilon_{r}+c_{12} \varepsilon_{\theta} \\
& \sigma_{\theta}=c_{21} \varepsilon_{r}+c_{22} \varepsilon_{\theta}
\end{aligned}
$$

where, $\varepsilon_{r}$ and $\varepsilon_{\theta}$ refer to the radial and circumferential direction strain tensor components.

For isotropic rock materials, Eq.(2) can be rewritten as

$$
\begin{aligned}
& \sigma_{r}=c_{1} \varepsilon_{r}+c_{2} \varepsilon_{\theta} \\
& \sigma_{\theta}=c_{2} \varepsilon_{r}+c_{1} \varepsilon_{\theta}
\end{aligned}
$$

where, $c_{1}=\frac{E(1-\mu)}{(1+\mu)(1-2 \mu)}$ and $c_{2}=\frac{E \mu}{(1+\mu)(1-2 \mu)}, E$ and $\mu$ are Young's modulus and Poisson's ratio of the rock masses, respectively .

The strain-displacement relations in terms of the radial displacement $u$ are

$\varepsilon_{r}=\frac{d u(r, t)}{d r}$ and $\varepsilon_{\theta}=\frac{u(r, t)}{r}$

The dynamic equilibrium equation can be expressed as follows

$\frac{d \sigma_{r}}{d r}+\frac{\sigma_{r}-\sigma_{\theta}}{r}=\rho \frac{d^{2} u(r, t)}{d t^{2}}$

Using Eqs(2),(4)and(5), the governing equation of radial displacement becomes

$\frac{r^{2} d^{2} u(r, t)}{d r^{2}}+r \frac{d u(r, t)}{d r}-u(r, t)=\frac{r^{2}}{c^{2}} \frac{d^{2} u(r, t)}{d t^{2}}$

where, $c^{2}=c_{1} / \rho$ and $\rho$ is the material density.

The internal pressure can be expressed as follows:

$\sigma_{r}(a, t)=p(t), u(a, 0)=0$ 
where, $\sigma_{r}(a, t)$ and $u(a, 0)$ are radial stress and initial displacement, respectively.

The radial dynamic displacement $u(r, t)$ can be decomposed into quasi static part $u_{s t}(r, t)$ and dynamic part $u_{d y}(r, t)$. The quasi static part $u_{s t}(r, t)$ meets the static equilibrium equations and boundary conditions, Dynamic part meets the dynamic balance equation and stress free boundary condition.

$$
u(r, t)=u_{s t}(r, t)+u_{d y}(r, t)=u_{s t}(r, t)+\sum_{i=1}^{\infty} U_{i}(r) \psi_{i}(t)
$$

Hydrostatic analysis on the quasi static problem. For the quasi static problem, radial and tangential stresses are determined using the elastic mechanical theory as follows

$$
\frac{d^{2} u_{s t}(r, t)}{d r^{2}}+\frac{1}{r} \frac{d u_{s t}(r, t)}{d r}+\left(\frac{-1}{r^{2}}\right) u_{s t}(r, t)=0
$$

Boundary conditions are:

$$
\sigma_{r}(a, t)=p(t), \quad \sigma_{r}(r, t)_{\mid r=\infty}=\sigma_{\infty}
$$

The quasi static displacement is

$$
u_{s t}(r, t)=\frac{r(1+\mu)}{E}\left(\sigma_{\infty}-p(t)\right)
$$

where, $p(t)$ is the internal pressure.

Dynamic analysis on the dynamic problem. Fluctuation model $U_{i}(r)$ can be obtained from the Eq.(12) [5]

$$
\frac{d^{2} U(r)}{d r^{2}}+\frac{1}{r} \frac{d U(r)}{d r}+\left(k^{2}-\frac{1}{r^{2}}\right) U(r)=0
$$

where, $\zeta=\frac{v}{1-v}$. Setting $k^{2}=\frac{\rho \omega^{2}}{c_{1}}$.

The boundary conditions are $\sigma_{r}(a, \omega)=0$ and $\sigma_{r}(r, \omega)_{\mid r>a}=0$.

where, $\omega^{2}$ is a nonnegative real number, the general solution is[6]

$$
U(r)=D \varepsilon_{\alpha}\left(\lambda r^{q}\right)
$$

where, $\varepsilon_{\alpha}\left(\lambda r^{q}\right)=\left(\beta_{1} J_{\alpha}\left(\lambda r^{q}\right)+\beta_{2} Y_{\alpha}\left(\lambda r^{q}\right)\right), q=1, \lambda=k, \alpha=1 . \beta_{1}$ and $\beta_{2}$ are unknown coefficients defined from the boundary conditions.

Eq. (3) can be written as

$$
\sigma_{r}(r, \omega)=c_{1} \frac{\partial U(r, \omega)}{\partial r}+c_{2} \frac{U(r, \omega)}{r}=\left(c_{1} \frac{\partial}{\partial r}+c_{2} r^{-1}\right) \times\left(\beta_{1} J_{\alpha}\left(\lambda r^{q}\right)+\beta_{2} Y_{\alpha}\left(\lambda r^{q}\right)\right)
$$

According to the quality of Bessel functions

$$
\begin{aligned}
& \frac{\partial}{\partial r}\left[J_{\alpha}\left(\lambda r^{q}\right)\right]=\left[J_{\alpha-1}\left(\lambda r^{q}\right)-\frac{\alpha}{\lambda r^{q}} J_{\alpha}\left(\lambda r^{q}\right)\right] \lambda q r^{q-1} \\
& \frac{\partial}{\partial r}\left[Y_{\alpha}\left(\lambda r^{q}\right)\right]=\left[Y_{\alpha-1}\left(\lambda r^{q}\right)-\frac{\alpha}{\lambda r^{q}} Y_{\alpha}\left(\lambda r^{q}\right)\right] \lambda q r^{q-1}
\end{aligned}
$$

Eq.(14) can be written as: 


$$
\begin{aligned}
& \sigma_{r}(r, \omega)=r^{-1}\left(-\alpha q c_{1}+c_{2}\right) \\
& {\left[\beta_{1} J_{\alpha}\left(\lambda r^{q}\right)+\beta_{2} Y_{\alpha}\left(\lambda r^{q}\right)\right]+c_{1} \lambda q r^{q-1}\left[\beta_{1} J_{\alpha-1}\left(\lambda r^{q}\right)+\beta_{2} Y_{\alpha-1}\left(\lambda r^{q}\right)\right] } \\
& \text { or: } \sigma_{r}(r, \omega)=\beta_{1} \Lambda_{1}(r)+\beta_{2} \Lambda_{2}(r)
\end{aligned}
$$

Eq.(18) takes the following form with boundary conditions:

$$
\left[\begin{array}{ll}
\Lambda_{1}(a, \omega) & \Lambda_{2}(a, \omega) \\
\Lambda_{1}(r, \omega)_{\mid r=\infty} & \Lambda_{2}(r, \omega)_{\mid r=\infty}
\end{array}\right]\left\{\begin{array}{l}
\beta_{1} \\
\beta_{2}
\end{array}\right\}=\left\{\begin{array}{l}
0 \\
0
\end{array}\right\}
$$

The simple form of Eq.(19) can be rewritten as follows

$$
\breve{\Lambda} \ddot{\beta}=\stackrel{\prime}{0}
$$

where, $\stackrel{\mathbf{\beta}}{\beta}$ is eigenvector and setting $|\Lambda|=0$.

Rao[7] had proved that elastomers have countless positive eigenvalues. The roots $\omega_{i}(i=1,2,3 \ldots)$ of Eq.(21) correspond to natural frequencies and the roots are all real and simple. Eq.(21) is calculated with Newton-Raphson method.

In the i-th fluctuation model, function $U_{i}(r)$ of $\omega_{i}$ can be written as follows

$$
\begin{aligned}
& U_{i}(r)=D_{i} \varepsilon_{\alpha}\left(\lambda_{i} r^{q}\right) \\
& \varepsilon_{\alpha}\left(\lambda_{i} r^{q}\right)=\left[\beta_{1 i} J_{\alpha}\left(\lambda_{i} r^{q}\right)+\beta_{2 i} Y_{\alpha}\left(\lambda_{i} r^{q}\right)\right]
\end{aligned}
$$

$\beta_{1}$ and $\beta_{2}$ are unknown coefficient corresponding to $\omega_{i}$, and also used in Eq.(19).

For the orthogonal condition

$$
\int_{a}^{\infty} \rho U_{i}(r) U_{j}(r) 2 \pi r d r=\delta_{i j}
$$

where, $\delta_{i j}$ is Kronecker function.

According to the orthogonal condition, the coefficient $D_{i}$ becomes

$$
\int_{a}^{\infty} \rho \varepsilon_{\alpha}\left(\lambda_{i} r^{q}\right) 2 \pi r d r=D_{i}
$$

From Eq.(7), Eq.(25) takes the following form

$$
\sum_{i=1}^{\infty}\left(\frac{d^{2} \psi_{i}(t)}{d t^{2}}+\omega_{i} \psi_{i}(t)\right) U_{i}(r)=-\frac{d^{2} u_{s t}(r, t)}{d t^{2}}
$$

According to the orthogonal condition, differential equation of $\psi_{i}(t)$ takes the following form

$$
\frac{d^{2} \psi_{i}(t)}{d t^{2}}+\omega_{i}^{2} \psi_{i}(t)=\frac{d^{2} \Phi_{i}(t)}{d t^{2}}
$$

where, $\Phi_{i}(t)=-\int_{a}^{\infty} \rho u_{s t}(r, t) U_{i}(r) 2 \pi r d r$.

According to the initial boundary condition, initial displacement and initial velocity can be written as follows

$$
u_{0}(r)=u_{s t}(r, 0)+\sum_{i=1}^{\infty} U_{i}(r) \psi_{i}(0)
$$




$$
v_{0}(r)=\frac{d u_{s t}(r, 0)}{d t}+\sum_{i=1}^{\infty} U_{i}(r) \frac{d \psi_{i}(0)}{d t}
$$

then,

$$
\begin{aligned}
& \psi_{i}(0)=\int_{a}^{\infty} \rho u_{0}(r) U_{i}(r) 2 \pi r d r+\Phi_{i}(0) \\
& \frac{d \psi_{i}(0)}{d t}=\int_{a}^{\infty} \rho v_{0}(r) U_{i}(r) 2 \pi r d r+\frac{d \Phi_{i}(0)}{d t}
\end{aligned}
$$

Finally,

$$
\begin{aligned}
& \psi_{i}(t)=\psi_{i}(0) \cos \left(\omega_{i} t\right)+\frac{1}{\omega_{i}} \frac{d \psi_{i}(0)}{d t} \sin \left(\omega_{i} t\right)+ \\
& \frac{1}{\omega_{i}} \int_{0}^{t} \frac{d^{2} \Phi_{i}(\tau)}{d \tau^{2}} \sin \left[\omega_{i}(t-\tau)\right] d \tau=\frac{d^{2} \Phi_{i}(\tau)}{d \tau^{2}} \frac{\left[1-\cos \left(t \omega_{i}\right)\right]}{\omega_{i}^{2}}
\end{aligned}
$$

After appropriate calculations, the following expressions for the displacement is written as

$$
u(r, t)=u_{s t}(r, t)+u_{d y}(r, t)=u_{s t}(r, t)+\sum_{i=1}^{\infty} U_{i}(r) \psi_{i}(t)
$$

Results and Analysis. Effects of parameters on the release of in-situ stress of surrounding rock mass around a circular tunnel are taken into account. Following computation parameters are used: in-situ stress of surrounding rock mass $\sigma_{\infty}=30 M P a$, Poisson's ratio $\mu=0.3$, Elastic modulus $E=3 G P a$, Radius of the tunnel $a=7 \mathrm{~m}$, density of surrounding rock mass $\rho=2650, t_{0}$ is the unloading completion time.

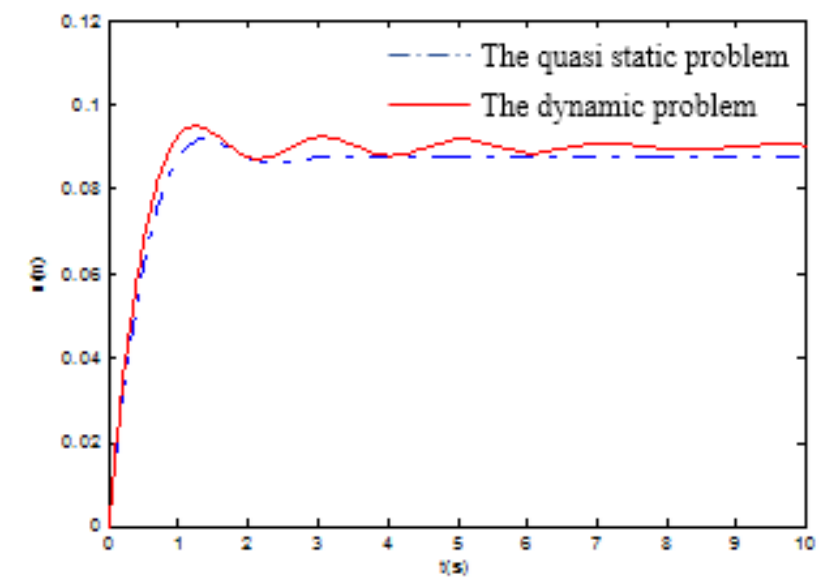

Fig. 3: Displacement curve of the interior the tunnel $\left(t_{0}=0.5 \mathrm{~s}\right)$

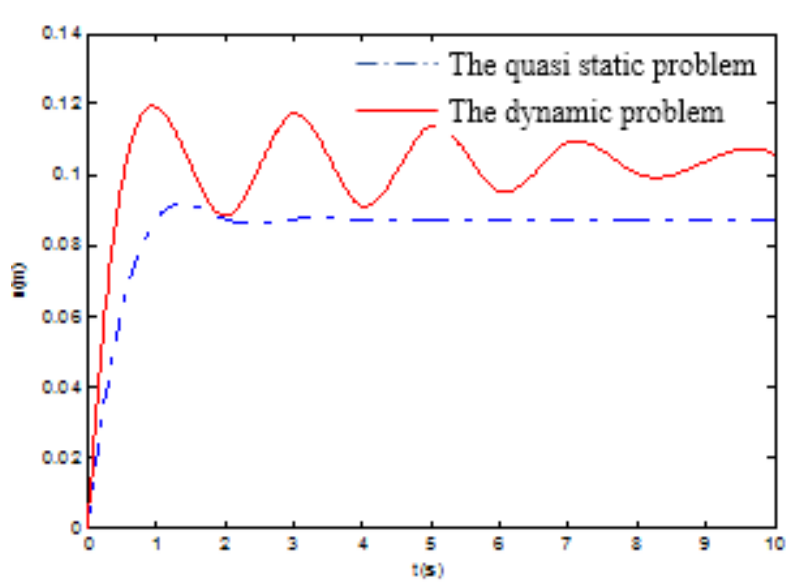

Fig. 4: Displacement curve of the interior wall of wall of the tunnel $\left(t_{0}=0.2 \mathrm{~s}\right)$

As shown in Figure 3 and Figure 4, the displacement of dynamic problem is bigger than that of quasi-static problem. The displacement of dynamic problem is time-sensitive, and then reached a steady condition finally.

The unloading completion time also has large effects on the displacement of dynamic problem. While, the displacement of quasi-static problem is not sensitive to the unloading completion time. Compared to Figure 3, as the rate of unloading decreases, the disturbance of the interior wall of the tunnel increases, as shown in Figure 4. 


\section{Conclusions}

A new mechanical model is proposed to investigate the dynamic response of the surrounding rock masses around a circular tunnel subjected to dynamic unloading. Effects of unloading rate and dynamic mechanical parameters of isotropic rock masses on dynamic response of the surrounding rock masses around a circular tunnel as well as the total elastic stress field distributions are taken into account. The numerical computation is made. It is found from numerical results that:

(1) The displacement of dynamic problem is bigger than that of quasi-static problem. The displacement of dynamic problem is time-sensitive, and then reached a steady condition finally.

(2) The unloading completion time also has large effects on the displacement of dynamic problem. While, the displacement of quasi-static problem is not sensitive to the unloading completion time.

(3) As the rate of unloading decreases, the disturbance of the interior wall of the tunnel increases.

\section{Acknowledgements}

The paper is supported by the Research Funds of Circular anti-slide pile force analysis and landslide treatment(ZL-2013-01)

\section{References}

[1]. W.B. Lu, J.H. Yang, P. Yan, M. Chen, Chuangbing Zhou, Yi Luo, Li Jin. (2012) Dynamic response of rock mass induced by the transient release of in-situ stress. International Journal of Rock Mechanics \& Mining Sciences, 53:129-141.

[2]. W.B.Lu, J. Li, M. Chen. (2005) Study on the mechanism of the loosing of the jointed rock mass caused by the dynamic unloading of initial stress during rock blasting. Chinese Journal of Rock Mechanics and Engineering, 24(1):4653 - 4657. [In Chines]

[3]. C.P. Yi, H.T. Xu, W.B. Lu. (2005) The dynamic unloading effect study of initial stress field in excavation process of large-scale rock mass structure. Chinese Journal of Rock Mechanics and Engineering, 24(1):4750-4784. [In Chines]

[4]. J.X. Ren, X.R. Ge, Y.B. Pu, et al. (2000) Primary study of real-time testing of unloading damage evolution law of rock. Chinese Journal of Rock Mechanics and Engineering, 19(6):697-701. [In Chines]

[5]. M. Shariyat. (2011) Exact and numerical elastodynamic solutions for thick-walled functionally graded cylinders subjected to pressure shocks. International Journal of Pressure Vessels and Piping. 88:75-87.

[6]. X.P. Zhou, Q.H. Qian. (2007) Zonal Fracturing Mechanism in Deep Tunnel. Chinese Journal of Rock Mechanics and Engineering, 5(26):877-885. [In Chinese]

[7]. S.S. Rao. (2007) Vibration of continuous systems. Wiley 